\title{
Ontario Ministry of Health and Long-Term Care Forecast for Hip Replacements
}

Pat Finerty

\section{Background}

Rapid growth in the need for orthopedic services is being experienced in Ontario and other provinces across Canada. Improvements in surgical technique, combined with an aging population, have fuelled dramatic increases in service demand for orthopedic surgery, raising concerns about the healthcare system's ability to meet the long-term demand - particularly for hip and knee procedures.

\section{Business Problem}

Between 1994 and 2004, hip replacement surgeries increased by $43 \%$ across Canada (Canadian Institute for Health Information 1996, 2006). In 2002, Ontario performed approximately 8,500 of these procedures, which represented $42 \%$ of the national demand of 20,000 cases (Canadian Joint Replacement Registry 2004). The increasing demand for hip-related surgery created pressures on orthopedic services, resulting in wait times becoming unacceptably long. A key factor contributing to the length of orthopedic wait times was the availability of orthopedic surgeons in Ontario.

The Ontario Ministry of Health and Long-Term Care found itself facing public expectations for improved service levels but lacking much of the knowledge about future hip surgery demands to plan its response strategy. Both patient and provider dissatisfaction had been publicly aired over lengthy wait times and limited system capacity (Hunter and Robertson 2001). Therefore, in 2004, the ministry developed its provin- cial strategy for wait times reduction for cancer care, cardiac services, cataract surgery, magnetic resonance imaging and computed tomography examinations and hip and knee replacements, announced by the provincial health minister at the time, George Smitherman (Ontario Ministry of Health and LongTerm Care 2004).

By early 2005, the ministry was confronting three issues that could inhibit its ability to effectively plan wait times improvements:

1. Retrospective data indicated a well-established demand trend for hip replacements, which had significantly increased during the prior three-year period.

2. Little reliable information was available about what the determinants of need for hip surgery were and how the interrelationship between these factors might influence future demand.

3. Reliable wait times information was not generally available since it was not usually collected. Even when collected, the lack of standards made data inconsistent and only marginally useful.

The ministry urgently needed a reliable method of forecasting future demand for hip replacements. Clearly, this could not be done by analyzing retrospective data alone, which could only offer crude regression indicators. The forecast would have to be scientifically derived and rigorous enough to guide Ontario's 
major strategic decisions on investments in medical manpower, facilities and services that would enable the province to achieve its performance mandate of reducing patient wait times for the identified procedures.

\section{Business Solution}

The ministry engaged SAS to examine the feasibility of developing an analytics forecast for hip replacement surgery over the next 14 years. SAS worked with the ministry to scope out a project to help predict individuals requiring hip replacement surgery by (1) developing an understanding of the primary determinants of need for hip replacements; (2) acquiring relevant data; (3) creating a forecast that would identify future demand for hip replacement surgery across Ontario over the next 14 years; and (4) documenting experiential learning from the hip replacement forecast project that would provide value to similar planning challenges in other health programs. SAS proposed a solution that became known as the Health Insight and Prediction Platform (HIPP).

\section{Methodology}

The HIPP solution is based on a four-step process: (1) data extraction, to create a raw file of relevant data extracted from multiple sources; (2) data mining, to derive a balanced subset of statistically significant data; (3) forecasting, to run the data against multiple mathematical models and testing the results for fit; and (4) reporting, to provide results to the client.

\section{Data Acquisition}

Three data sources were selected as most relevant to the project. The following data were extracted for the previous 10-year period:

1. Physician billing records. Claims data from hip surgeryrelated procedures were used to derive information about the onset of hip disease and referral for surgery. These data were drawn from procedure and diagnostic coding and events that could be determined from the fee code, sourced from the Ontario Health Insurance Plan.

2. Acute care admission/separation records. These data were used to acquire details of procedures carried out in Ontario hospitals. In-patient and day-procedure records provided hip replacement surgery data based on hospital stays and day-procedures, as well as data derived from the World Health Organization (1979) International Classification of Diseases, Ninth Revision, diagnostic and service utilization codes (sourced from the Canadian Institute for Health Information).

3. Statistics Canada population forecasts. These data were used to provide census-based projections of the age, sex and demographics of the Ontario geographical population (sourced from Statistics Canada).

In addition, the Ontario Health Registered Persons file (sourced from the ministry and devoid of personal information) was used for accurate, non-attributable linkage of patientphysician and patient-procedure relationships. In all, the data set contained over 33,000 observations using 67 variables. Statistics Canada population demographics were then overlaid based on patient geographical Canada Post forward sortation area (FSA) coding.

\section{Data Mining}

The linked data set was screened to eliminate those cases that had insufficient, inconsistent or missing information or were outside the sample age window ( 40 years old at year 0 of the time scale). Subsequently, cross-correlations were run between all variables to uncover previously unknown data relationships. This provided valuable insight into the variables that would ultimately be best suited to increase forecasting accuracy. SAS data set-editing tools were employed to prepare the data for statistical analysis. Oversampling was used to select a balanced data set for time series analysis. Missing data were resolved so that models requiring data integrity, such as neural networks and regression techniques, could be used reliably. Variables with extreme outlying values were replaced or removed during the mining process. Finally, those values that were found to have a highly skewed distribution were adjusted through mathematical transformation to make the data set suitable for advanced statistical analysis.

\section{Forecasting}

Using tests such as autocorrelation and white noise, the data were validated for modelling suitability. The forecast modelling process then ran a number of different time series modelling techniques to identify which produced the best demand forecast for hip replacement surgery. These included Box-Jenkins, autoregressive integrated moving average (ARIMA) models, exponential smoothing models, intermittent demand models and unobserved components models.

Each model was assessed on the accuracy of the forecast against a holdout data set, the statistical error of the forecast and statistical measures of accuracy such as mean absolute percent error (MAPE). A manually generated ARIMA model was finally adopted as the most reliable and was run against the full-time series.

\section{Reporting}

At the conclusion of this forecasting project, a number of reports were delivered to the client, including the following: 
- A series of graphic time plots of the forecast, along with graphic illustrations of model behaviour

- A tabular forecast by month, to 2020 , detailing the forecasted incidence of hip surgery, along with confidence intervals, standard error and other key descriptive qualifiers

- A detailed SAS consulting report containing (1) a methodological description of the total process; (2) a significant amount of collateral information on the data set; (3) a review of the relevance or otherwise of various models; and (4) descriptive information on the data set with the results of cross-correlations and prediction error calculations of most of the 67 variables

Building on a review of the HIPP methodology and results, the ministry was then able to refine its forecasting requirements and develop long-term models for hip and knee surgery forecasting.

\section{HIPP Solution Overview}

The HIPP is a combination of statistical techniques and tools. When applied along with health system contextual knowledge and data, it offers a powerful solution to help predict the incidence of many disease situations. The principal tool set for HIPP is the SAS Forecasting Studio, a component of the SAS Enterprise Intelligence Platform, which includes SAS Data Integration, Intelligence Storage, Analytic Intelligence and Business Intelligence. For more information on SAS products and solutions, visit www.sas.com.

\section{References}

Canadian Institute for Health Information. 1996. Hospital Morbidity Database, 1994-1995. Ottawa: Author.

Canadian Institute for Health Information. 2006. Hospital Morbidity Database, 2004-2005. Ottawa: Author.

Canadian Joint Replacement Registry. 2004. 2004 Report Total Hip and Total Knee Replacements in Canada. Ottawa, ON: Canadian Institute for Health Information.

Hunter, D. and D. Robertson. 2001. “Total Hip Replacement Needs Far Exceed Supply." Canadian Medical Association Journal 165(4): 395.

Ontario Ministry of Health and Long-Term Care. 2004. McGuinty Government Reduces Wait Times for Key Surgeries. Toronto, ON: Government of Ontario. Retrieved February 12, 2009. <http://ogov.newswire.ca/ontario/GPOE/2004/12/14/c5113. html? lmatch=\&lang=_e.html>.

World Health Organization. 1979. International Classification of Diseases, Ninth Revision. Geneva, Switzerland: Author.

\section{About the Author}

Pat Finerty is a vice-president at SAS Canada, Toronto, Ontario, and is responsible for leading the development of new industry verticals, practice areas and business models to foster the Canadian subsidiary's long-term growth. 